\title{
Below Band Gap Formation of Solvated Electrons in Neutral Water Clusters?
}

\section{Journal Article}

\section{Author(s):}

Ban, Loren (1); West, Christopher W.; Chasovskikh, Egor; Gartmann, Thomas E.; Yoder, Bruce L.; Signorell, Ruth (i)

\section{Publication date:}

2020-10-01

\section{Permanent link:}

https://doi.org/10.3929/ethz-b-000439029

\section{Rights / license:}

Creative Commons Attribution-NonCommercial-NoDerivatives 4.0 International

\section{Originally published in:}

The Journal of Physical Chemistry A 124(39), https://doi.org/10.1021/acs.jpca.0c06935

\section{Funding acknowledgement:}

786636 - Droplet Photoelectron Imaging (EC)

172472 - Phase Transitions of Ultrafine Aerosol Particles: Condensation, Freezing, and Metal Formation in Confined Systems (SNF)

801459 - Fellowship Program of the NCCR MUST (National Competence Center for Research in Molecular Ultrafast Science and Technology) and the Cluster of Excellence RESOLV (EC) 


\title{
Below Band Gap Formation of Solvated Electrons in Neutral Water Clusters?
}

\author{
Loren Ban, Christopher W. West, Egor Chasovskikh, Thomas E. Gartmann, Bruce L. Yoder, \\ and Ruth Signorell*
}

Cite This: J. Phys. Chem. A 2020, 124, 7959-7965

Read Online

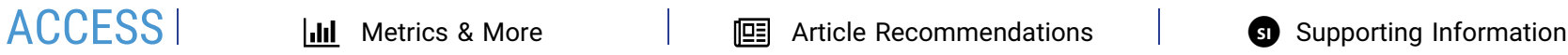

ABSTRACT: Below band gap formation of solvated electrons in neutral water clusters using pump-probe photoelectron imaging is compared with recent data for liquid water and with above band gap excitation studies in liquid and clusters. Similar relaxation times on the order of $200 \mathrm{fs}$ and $1-2$ ps are retrieved for below and above band gap excitation, in both clusters and liquid. The independence of the relaxation times from the generation process indicates that these times are dominated by the solvent response, which is significantly slower than the various solvated electron formation processes. The analysis of the temporal evolution of the vertical electron binding energy and the electron binding energy at half-maximum suggests a dependence of the solvation time on the binding energy.

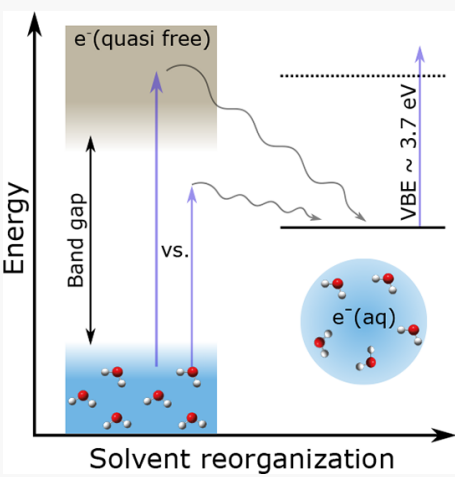

\section{INTRODUCTION}

Solvated electrons in molecular liquids, especially water, have sparked broad interest because of their widespread occurrence and their intriguing fundamental properties. Numerous experimental and theoretical studies have provided insights into the electronic properties, the generation mechanism, and the relaxation dynamics of solvated electrons in liquid water, amorphous ice, anionic water clusters, neutral water clusters, and sodium-doped water clusters ${ }^{1-48}$ (and references therein).

A series of comparable, femtosecond time-resolved photoelectron studies on liquid water and neutral water clusters ${ }^{49-51}$ has now opened up the possibility to address two aspects in greater detail: (i) similarities and differences of the relaxation dynamics after below versus above band gap generation of the hydrated electron and (ii) similarities and differences of the relaxation dynamics in two different aqueous environments, i.e., liquid water versus neutral water clusters. The formation of hydrated electrons by irradiation with photons has been observed down to the absorption edge of water at $\sim 6$ $\mathrm{eV}^{37-39,52}$ (and references therein), far below the bottom of the conduction band. ${ }^{53-56}$ Different mechanisms have been suggested for the below band gap formation of solvated electrons, involving very fast $(<10 \mathrm{fs})$ water dissociation and proton and electron transfer processes ("hot $\mathrm{H}$ atom mechanism", "proton-coupled electron transfer", "consecutive proton transfer, electron transfer process", "electron transfer to preexisting sites"; see refs $37-39$ and 52 and references therein). These ultrafast processes are followed by slower solvent rearrangement (fs to ps time scales) and slow geminate recombination $^{37,40,47,57-59}$ (and references therein). Irradia- tion with photon energies above the band gap, by contrast, produces delocalized, quasi-free conduction band electrons by the ionization of water. These conduction band electrons relax rapidly toward the bottom of the conduction band by fast energy dissipation through electron scattering on time scales of several 10 fs. ${ }^{15,31,60-63}$ Localization from the bottom of the conduction band to interband trapped states likely takes place on time scales faster than $\sim 100 \mathrm{fs},{ }^{15,25,30,34}$ followed by slower solvent rearrangement and geminate recombination.

The present time-resolved photoelectron imaging study addresses the question of whether the formation of solvated electrons by below band gap excitation is also feasible in neutral water clusters, as previously observed in liquid water $^{38,50}$ (and references therein). The relaxation dynamics after one-photon excitation at $7.8 \mathrm{eV}$ photon energy is probed and compared with a recent pump-probe study for liquid water using an excitation energy of $7.7 \mathrm{eV} .^{50}$ At these excitation energies, it is assumed that solvated electron formation follows absorption into a localized $1^{1} \mathrm{~B}_{1}$ excited state of water. The results for below band gap excitation are compared with previous above band gap excitation photoelectron studies for the liquid and clusters. ${ }^{49-51}$

Received: July 29, 2020

Revised: September 1, 2020

Published: September 2, 2020 


\section{METHODS}

The velocity map imaging (VMI) photoelectron spectrometer used in this work is described in refs $36,49,64$, and 65 and the Supporting Information (SI). Neutral water clusters consisting of $\sim 500$ water molecules $(r \sim 1.5 \mathrm{~nm}$, see refs 64 and 66 for cluster size determination) are generated in supersonic expansions. Femtosecond (fs) laser pulses of $7.8 \mathrm{eV}$ photon energy (pump) from high harmonic generation (HHG) are used to generate solvated electrons by below band gap excitation in water clusters. The relaxation dynamics are probed with a femtosecond probe pulse of $4.7 \mathrm{eV}$ photon energy by varying the pump-probe time delay $t$. Timeresolved electron binding energy spectra $(\mathrm{eBE})$ and photoelectron angular distributions (PADs, $\beta$-parameters eq S1, SI) are retrieved from the recorded time-dependent photoelectron images (SI). The instrument response function (IRF) determined from $\left(1+1^{\prime}\right)$ nonresonant ionization of $\mathrm{Xe}$ is $150 \pm 35$ fs.

\section{RESULTS}

Figure 1a shows time-resolved photoelectron spectra (TRPES) recorded with a $4.7 \mathrm{eV}$ probe pulse following below band gap

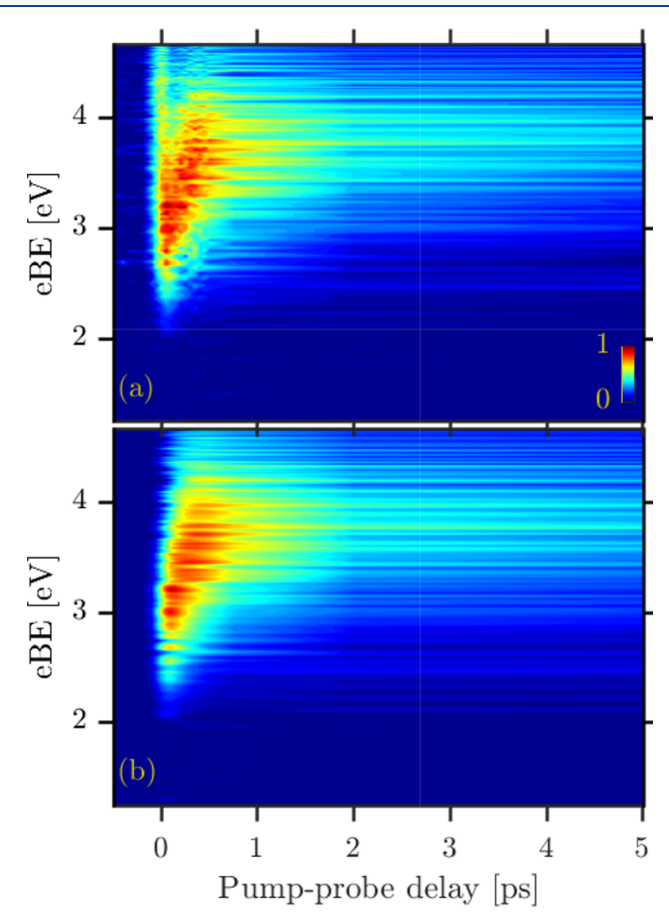

Figure 1. Time-resolved photoelectron spectra (TRPES) of the hydrated electron in neutral water clusters. (a) Experimental TRPES measured with a $7.8 \mathrm{eV}$ pump pulse and a $4.7 \mathrm{eV}$ probe pulse (without subtraction of the impulsive component). (b) Global Lifetime Analysis (GLA) fit to the TRPES in panel a after subtraction of the impulsive component at early pump-probe delays (see also Figure S1).

excitation at $7.8 \mathrm{eV}$ pump energy. Within the first picosecond, the eBE spectrum changes rapidly and approaches an asymptote at longer times, accompanied by a decrease in the overall signal intensity. The observed spectral signature is characteristic of hydrated electron relaxation dynamics, where the fully relaxed hydrated electron in its ground state is formed after $\sim 2 \mathrm{ps}^{49-51}$ followed by depopulation due to geminate recombination on a much longer time scale. ${ }^{50}$ This confirms hydrated electron formation in neutral clusters following below band gap excitation.

We expect the short-lived signal observed around zero time delay extending toward maximum eBEs of $\sim 4.7 \mathrm{eV}$ (toward zero photoelectron kinetic energy, eKE) to originate either from the fast dynamics (much faster than our IRF) of the background water vapor ${ }^{67}$ or the impulsive $\left(1+1^{\prime}\right)$ ionization of water clusters. To subtract these spectral components, we performed a Global Lifetime Analysis (GLA) of our data. ${ }^{18,68,69}$ GLA assumes a number of time-independent spectra and assigns them a corresponding exponentially decaying population. The spectral profiles and the decay dynamics are then fit to the experimental data. This technique has recently been applied by Hara et al. ${ }^{69}$ to model detailed kinetics following conduction-band excitation of methanol. Since the relaxation dynamics are faster in water than in methanol, ${ }^{50,69,70}$ much better time resolution than that currently available and excellent signal-to-noise levels would be required to extract similarly detailed kinetic information for aqueous systems. We thus use here a simple sequential kinetics model (eq S2, SI) with three exponentially decaying features and a Gaussian distribution for the IRF to fit the TRPES in Figure 1a (SI, section S2), similar to the approaches in refs 50 and 69 . The best TRPES fit (Figure $1 \mathrm{~b}$ and Figure S1, middle panel) corresponds to a Gaussian with a full-width at halfmaximum $\mathrm{FWHM}_{\mathrm{IRF}}=166 \pm 5 \mathrm{fs}$, consistent with the experimentally determined IRF of $150 \pm 35 \mathrm{fs}$, and three exponential decays with lifetimes of $\tau_{1}^{\mathrm{GLA}}=221 \pm 56 \mathrm{fs}, \tau_{2}^{\mathrm{GLA}}=$ $447 \pm 100 \mathrm{fs}$, and $\tau_{3}^{\mathrm{GLA}}=17.74 \pm 5.55 \mathrm{ps}$ (SI and Figure 2) with the corresponding Decay Associated Spectra (DAS;
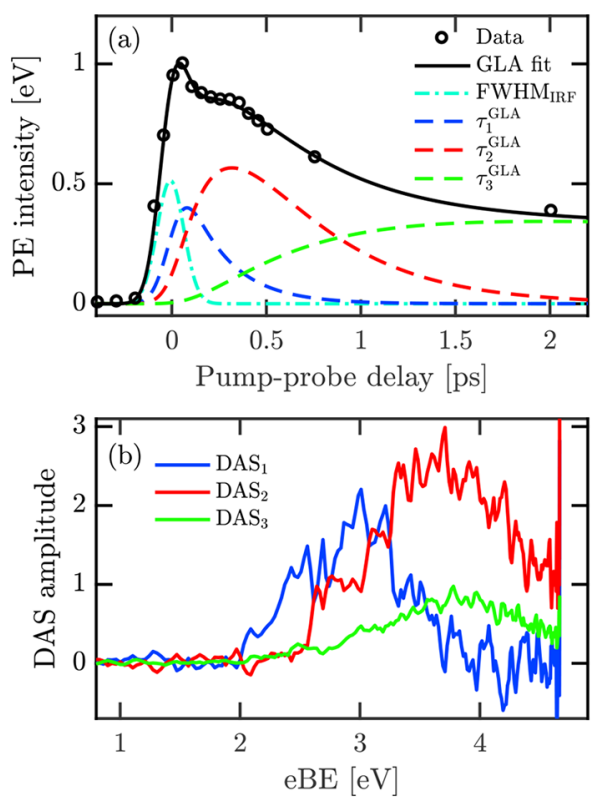

Figure 2. (a) Experimental (circles) and fitted (full black line) timedependent relative photoelectron yield with the contributions from the corresponding decay associated spectra (DAS, dashed colored lines). The Gaussian component (cyan dashed-dotted line, $\mathrm{FWHM}_{\mathrm{IRF}}$ $=166 \pm 5 \mathrm{fs}$ ) matches well with the measured instrument response function. Contributions from $\mathrm{DAS}_{1}$ (blue dashed line, $\tau_{1}^{\mathrm{GLA}}=221 \pm$ $56 \mathrm{fs}$ ), $\mathrm{DAS}_{2}$ (red dashed, $\tau_{2}^{\mathrm{GLA}}=447 \pm 100 \mathrm{fs}$ ) line, and $\mathrm{DAS}_{3}$ (green dashed line, $\tau_{3}^{\mathrm{GLA}}=17.74 \pm 5.55 \mathrm{ps}$ ) follow a sequential kinetics mechanism. (b) The three DAS obtained from the fit (SI, section S2). $\mathrm{DAS}_{1}$, blue line; $\mathrm{DAS}_{2}$, red line; $\mathrm{DAS}_{3}$, green line. 
Figure $2 \mathrm{~b}$ ). This analysis allows us to subtract the impulsive component from the TRPES (Figure $1 b$, see also eBE spectra in Figures S3 and S4).

Figure 2a shows the time-dependent, relative photoelectron yield of the hydrated electron (experimental data: circles, GLA fit: full black line) with the contributions from the corresponding decay components (dashed colored lines). The hydrated electron yield decays to $\sim 45 \%$ after 2 ps and to $\sim 33 \%$ after 5 ps of the yield measured at $200 \mathrm{fs}$. Within our uncertainties $( \pm 15 \%)$, these values are comparable to the measurements in liquid water of $\sim 31 \%$ and $\sim 22 \%$, respectively, ${ }^{50}$ hinting at similar loss mechanisms in clusters and liquid, at least for these relatively short pump-probe delay range. Electron yields recorded at long pump-probe time delays have previously been used to extract geminate recombination rates, survival probabilities, and retrieved electron ejection lengths in the liquid $^{50}$ (and references therein). In clusters, it is difficult to obtain reliable experimental data for long pump-probe delays because of the generally lower signal-to-noise level.

Experimental time-dependent eBE spectra are shown before subtraction of the impulsive component in Figure S2 and after subtraction of the impulsive component in Figure S3. Fitting these spectra with an exponentially modified Gaussian function (eq S6 and Figures S2 and S3) allows us to retrieve the time evolution of the vertical electron binding energy (VBE; most probable $\mathrm{eBE}$ ) and the electron binding energy at halfmaximum (HBE; see below). At short time delays $<60 \mathrm{fs}$, subtraction of the impulsive component is required to obtain reliable VBEs, while the VBEs at longer time delays are indistinguishable within our experimental uncertainty regardless of whether the impulsive component is subtracted or not (Figures S2 and S3). The experimental VBEs at time delays $>60 \mathrm{fs}$ are shown in Figure 3a (circles). The VBE shifts from an initial value of 3.0 to $3.75 \mathrm{eV}$, which is reached at $\sim 2$ ps and remains constant afterward. The eBE spectra with a VBE of $3.75 \mathrm{eV}$ recorded after $\sim 2$ ps (Figures S2 and S3) coincide with those of the relaxed ground state hydrated electron recorded in clusters and the liquid at a probe energy of 4.7 $\mathrm{eV}^{2,13,31,46,49,71}$ We have also performed cluster size-dependent studies for average cluster sizes between $\langle n\rangle \sim 250$ and 500 molecules. No cluster size-dependence of the VBE (at $\sim 2$ ps) is observed within the experimental uncertainty (see ref 49 for comparison with water anion and $\mathrm{Na}$-doped water clusters). In agreement with our previous study using 10.9 $\mathrm{eV}$ pump photons, ${ }^{49}$ we retrieve a $\beta$-parameter of $\sim 0.2$ for the relaxed ground state hydrated electron (after $\sim 2 \mathrm{ps).} \mathrm{After}$ scattering corrections, ${ }^{31,49,61,64}$ this corresponds to a genuine $\beta$-parameter in the range of $0.51-0.66$. For the present pump photons of $7.8 \mathrm{eV}$, no significant time-dependence is observed for the $\beta$-parameter, i.e., the value of $\beta$ lies around $\sim 0.2$ for all pump-probe delays. This contrasts with our previous results using $10.9 \mathrm{eV}$ pump photons, where the initial $(t=0 \mathrm{fs}) \beta$ parameter of $\sim 0.4$ exceeded the ground state value of $\sim 0.2 .{ }^{49}$ However, such differences must be viewed with caution given our relatively high experimental uncertainty of $\sim \pm 0.15$.

\section{DISCUSSION}

The temporal evolution of the VBE provides data on the relaxation dynamics following below band gap excitation and allows us to compare them with data from previous studies. We find that the time-dependence of the VBEs in Figure 3a (black circles) is well represented by a biexponential function (black
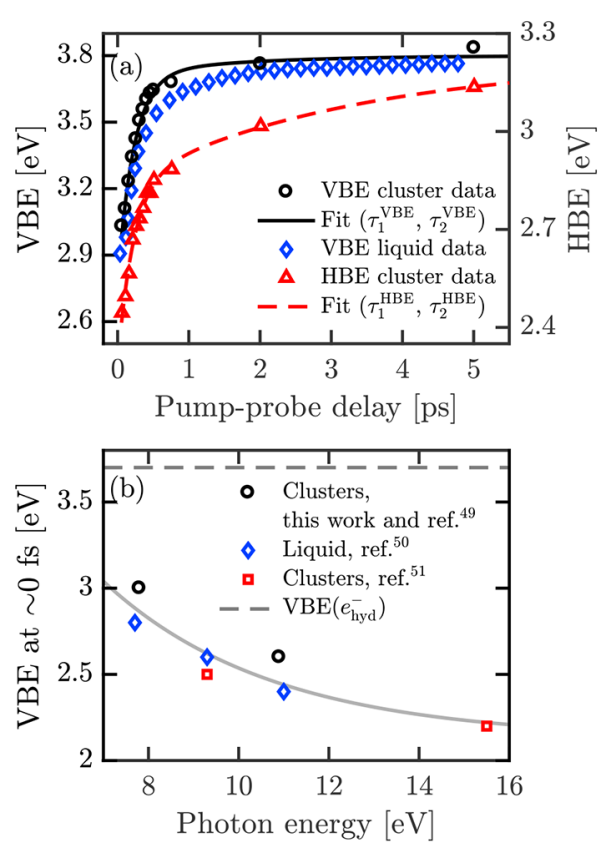

Figure 3. (a) Time-dependent vertical binding energy (VBEs, black circles, left abscissa) and binding energy at half-maximum (HBEs, red triangles, right abscissa) for neutral water clusters recorded at a pump energy of $7.8 \mathrm{eV}$. The data are extracted from the TRPES in Figure 1 (see text and SI). The biexponential fit to the VBEs with time constants $\tau_{1}^{\mathrm{VBE}}=220 \mathrm{fs}$ and $\tau_{2}^{\mathrm{VBE}}=1.6 \mathrm{ps}$ is shown as the black full line. The biexponential fit to the HBEs with time constants $\tau_{1}^{\mathrm{HBE}}=220$ fs and $\tau_{2}^{\mathrm{HBE}}=3.3 \mathrm{ps}$ is shown as the red dashed line. Blue diamonds: time-dependent VBEs for liquid water extracted from Figure $2 \mathrm{~d}$ of ref 50 recorded at a pump energy of $7.7 \mathrm{eV}$. (b) VBE at a pump-probe delay of $\sim 0 \mathrm{fs}(\operatorname{VBE}(\mathrm{t} \sim 0 \mathrm{fs})$, see Table 1$)$ for the liquid from $\operatorname{ref}^{50}$ (blue triangles) and different cluster studies (black circles: this work and ref., ${ }^{49}$ red squares: ref $^{51}$ ) as a function of the pump energy $h v$. The gray line is to guide the eye. The horizontal dashed line at $\sim 3.75$ $\mathrm{eV}$ indicates the VBE of the relaxed ground state hydrated electron $\left(e_{\text {hyd }}^{-}\right)$which is reached after $\sim 2$ ps. Note that the different studies use somewhat different analysis methods and different time resolutions.

full line): $\operatorname{VBE}(t)=a_{1} \exp \left(-t / \tau_{1}^{\mathrm{VBE}}\right)+a_{2} \exp \left(-t / \tau_{2}^{\mathrm{VBE}}\right)+$ $\operatorname{VBE}(t=\infty)$, with $\operatorname{VBE}(t=\infty)=3.8 \mathrm{eV}$ and time constants $\tau_{1}^{\mathrm{VBE}}=220 \mathrm{fs}$ and $\tau_{2}^{\mathrm{VBE}}=1.6 \mathrm{ps}$. These time constants agree well (see Table 1) with those reported in a recent liquid microjet study of water by Suzuki and co-workers, ${ }^{50}$ following below band gap excitation using a very similar pump energy of $7.7 \mathrm{eV}$ (blue diamonds in Figure 3a). A similarly good agreement between the relaxation times of clusters and liquid was also observed at a pump energy of $9.3 \mathrm{eV}$ (Table 1). The former time scale $\left(\tau_{1}^{\mathrm{VBE}}=220 \mathrm{fs}\right)$ has been assigned to fast solvent reorganization and electron localization into a cavity with size on the order of a few $\AA$, which is further stabilized by slow solvent reorganization $\left(\tau_{2}^{\mathrm{VBE}}=1.6 \mathrm{ps}\right) .{ }^{8,30,34}$ Very similar time scales are also retrieved from the sequential kinetics model (Figure $2 \mathrm{~b}$ ). The relaxed hydrated electron $\left(\mathrm{DAS}_{3}\right.$, green line) is formed in $<2 \mathrm{ps,} \mathrm{through} \mathrm{the} \mathrm{transient} \mathrm{states}$ represented by $\mathrm{DAS}_{1}$ (blue line) and $\mathrm{DAS}_{2}$ (red line). The shorter time constant $\left(\tau_{1}^{\mathrm{GLA}}=221 \pm 56 \mathrm{fs}\right)$ is associated with the $\mathrm{DAS}_{1}$ evolving into $\mathrm{DAS}_{2}$ with a VBE shift of about $0.7 \mathrm{eV}$, consistent with the fast solvent reorganization $\left(\tau_{1}^{\mathrm{VBE}}=220 \mathrm{fs}\right)$. The evolution of $\mathrm{DAS}_{2}$ into $\mathrm{DAS}_{3}$ shows only a minor shift in the VBE (slow solvent reorganization) and significant loss of signal due to geminate recombination. However, this comparison is only qualitative since $\mathrm{DAS}_{1}$ and $\mathrm{DAS}_{2}$ only 
Table 1. Comparison of the Time Constants $\tau_{1}^{\mathrm{VBE}}$ and $\tau_{2}^{\mathrm{VBE}}$ for the Solvation of the Hydrated Electron in Water Clusters and Liquid Water Retrieved from the Time Evolution of the VBEs ${ }^{c}$

\begin{tabular}{lccccccc} 
& Liquid $^{50}$ & Cluster (this work) & Cluster $^{51}$ & Liquid $^{50}$ & Cluster $^{49}$ & Liquid (two-photon) $^{50}$ & Cluster $^{51}$ \\
\multicolumn{1}{c}{$\boldsymbol{h} \boldsymbol{v}[\mathrm{eV}]$} & 7.7 & 7.8 & 9.3 & 9.3 & 10.9 & 11.0 & 15.5 \\
$\boldsymbol{\tau}_{1}^{\mathrm{VBE}}[\mathrm{ps}]$ & 0.2 & 0.2 & 0.21 & 0.3 & & 0.2 & 0.18 \\
$\boldsymbol{\tau}_{2}{ }^{\mathrm{VBE}}[\mathrm{ps}]$ & 1.0 & 1.6 & 1.4 & 0.9 & & 2.0 & 1.3 \\
$\operatorname{VBE}(\mathrm{t} \sim 0 \mathrm{fs})[\mathrm{eV}]$ & 2.8 & 3.0 & 2.5 & $2.6^{a}$ & 2.6 & $2.4^{a}$ & $2.2^{b}$
\end{tabular}

${ }^{a}$ Values estimated from Figure 2e,f in ref 50. ${ }^{b}$ Value estimated from Figure S6c in ref 51. ${ }^{c} h v$ is the pump photon energy. Except for the data at 11 $\mathrm{eV}$, all excitations are single-photon excitations. $\mathrm{VBE}(\mathrm{t} \sim 0 \mathrm{fs})$ is the vertical binding energy at a pump-probe delay of $\sim 0$ fs. Note that the different studies use somewhat different analysis methods and different time resolutions.

contain information about the average spectrum of all transient species probed within our IRF.

Considering that $\tau_{1}^{\mathrm{VBE}}$ and $\tau_{2}^{\mathrm{VBE}}$ are likely dominated by the response of the solvent (see introduction and ref 15), the similarity of clusters and liquid does not appear so obvious. Clusters have greater surface area and are thus structurally less restricted than the liquid. One would thus expect solvent rearrangement to be faster in the cluster than in the liquid. The cluster temperatures are likely lower than those of the liquid, which would result in the opposite effect on the relaxation times, i.e., one would expect an increase of the solvent rearrangement times in the cluster compared with the liquid. Furthermore, one would expect both effects to be more pronounced for the slower solvent motions (larger amplitude reorientation), i.e., more pronounced for $\tau_{2}^{\mathrm{VBE}}$ than for $\tau_{1}^{\mathrm{VBE}}$. This appears to be the case for the data at 7.8 and $9.3 \mathrm{eV}$ (see Table 1). The slightly higher values of $\tau_{2}^{\mathrm{VBE}}$ in the clusters might imply that the cluster temperature is the dominant effect. However, such a conclusion remains speculative with the caveat of the limited comparability of cluster data with the liquid jet results. Apart from the somewhat different analysis methods used, $\tau_{2}^{\mathrm{VBE}}$ is generally less well constrained by the cluster data with their lower signal-to-noise and fewer data points at longer time delays.

The observed independence of $\tau_{1}^{\mathrm{VBE}}$ and $\tau_{2}^{\mathrm{VBE}}$ on the pump energy used (see data for the different $h v$ in Table 1 ) supports the conclusions that these time scales are dominated by solvent response. The generation process will mainly influence the very fast processes (e.g., electron scattering, localization from the conduction band into the band gap, the different below band gap formation mechanisms) taking place on time scales of a few tens of fs, while the generally slower solvent responses will at most weakly depend on the generation process. The fast component of the solvent relaxation would still be expected to correlate to some extent with electronic deactivation and localization so that it might come as a surprise to find almost identical values for $\tau_{1}^{\mathrm{VBE}}$ at all different pump energies (Table 1). However, one needs to keep in mind that these values lie close to the time resolution (on the order of $\sim 100 \mathrm{fs}$ ) of the studies. To properly access these fast processes, one would need a significantly better time resolution by about a factor of $\sim 10$. Observable effects of using different excitation energies are mainly limited to the PADs and VBEs at zero pump-probe delay $(t \sim 0 \mathrm{fs})$. As mentioned above, the initial $\beta$-parameter recorded at $10.9 \mathrm{eV}$ appears to be higher $(\sim 0.4)$ than the one measured at $7.8 \mathrm{eV}(\sim 0.2)$. Similarly, the initial $\operatorname{VBE}(\operatorname{VBE}(t \sim$ $0 \mathrm{fs})$ ) seems to decrease systematically with increasing pump energy by about $0.7 \mathrm{eV}$ (Figure $3 \mathrm{~b})$. At $7.8 \mathrm{eV}, \operatorname{VBE}(t \sim 0 \mathrm{fs})$ lies $\sim 0.7 \mathrm{eV}$ below the values for the relaxed ground state of the hydrated electron (horizontal dashed line at $\sim 3.75 \mathrm{eV}$ ), while this difference increases to $\sim 1.4$ at $15.5 \mathrm{eV}$ pump energy.
Together with the above information, this suggests that fast electronic relaxation processes remain largely hidden within the time resolution. As a result, different pump-probe studies in Table 1 probe very similar solvation dynamics probably starting from similar localized initial states in the band gap, independently of the pump energy.

The temporal evolution of the VBE is a simple representation of the complex underlying dynamics because it only probes the dynamics of states that correspond to the maximum of the eBE spectrum (Figure S2). In Figure 3a, we also show the time-dependence of the binding energy at halfmaximum (HBE, red triangles) on the rising edge of the eBE spectrum together with a biexponential fit (red dashed line), resulting in time constants of $\tau_{1}^{\mathrm{HBE}}=220 \mathrm{fs}$ and $\tau_{2}^{\mathrm{HBE}}=3.3 \mathrm{ps}$. $\tau_{2}^{\mathrm{HBE}}$ exceeds $\tau_{2}^{\mathrm{VBE}}$ by a factor of 2 . The HBE data represents the temporal evolution of a population with a lower binding energy compared with the population represented by the VBE data. This implies a correlation between the binding energy and the relaxation time. A potential explanation for this correlation could be that states with lower binding energy require more time to relax because more extensive solvent rearrangement is needed to reach the relaxed ground state. To represent this behavior, we therefore suggest using the $\mathrm{HBE}$ in addition to the VBE. A comparison with previous studies is currently not possible because the temporal evolution of the HBE is not available from those studies. Finally, we would like to highlight another advantage of the HBE when comparing different studies. Many of the solvated electron photoelectron studies use ultraviolet probe photons in the range below 5.8 $\mathrm{eV}$; i.e., in a range where the energy dependence of electron scattering has a pronounced influence on the shape of the measured binding energy spectra. ${ }^{2,31}$ Figure 3 in ref 31 shows that the position of the HBE only shifts by $50 \mathrm{meV}$ when changing the probe photons from 4.4 to $5.8 \mathrm{eV}$, while the position of the VBE shifts by more than $200 \mathrm{meV}$ over the same probe energy range. The HBE is less affected by the influence of scattering than the VBE and thus makes comparisons between studies using different probe wavelengths more reliable. We would like to emphasize that for solvated electrons in water, the preferred method to correct for such biases arising from electron scattering are detailed scattering simulations to extract genuine binding energy spectra. ${ }^{3,61-64,72}$ However, in most studies, this correction has not yet been implemented.

\section{CONCLUSIONS}

We have investigated the relaxation dynamics of the solvated electron in neutral water clusters ( $\sim 500$ molecules) after below band gap excitation with photons of $7.8 \mathrm{eV}$ using ultrafast photoelectron imaging. The comparison with a recent liquid water study at an excitation energy of $7.7 \mathrm{eV}^{50}$ reveals similar 
solvation times on the order of $\sim 200$ fs and $\sim 1-2$ ps in the clusters and the liquid. It has been suggested that at these excitation energies, hydrated electrons are generated by water dissociation and proton and electron transfer processes ${ }^{38}$ after one-photon absorption to the predominately localized $1^{1} B_{1}$ excited state in water. The comparison of the below band gap investigations with previous above band gap photoelectron studies ${ }^{4-51}$ shows that the observable relaxation dynamics are essentially independent of the excitation energy. This is not surprising considering the time scales accessible in these experiments $(\sim 100 \mathrm{fs}$ range $)$ that mainly probe the solvent response, which is largely independent of the generation process of the solvated electron. A much higher timeresolution (by at least better than a factor of 10) would be required to detect excitation energy dependent differences in the generation process of the solvated electron, arising from ultrafast processes, such as electron scattering, localization into the band gap, and different below band gap electron formation mechanisms. Finally, the different solvation dynamics observed for the binding energy at half-maximum and the vertical electron binding energy suggest that states with a lower electron binding energy correlate with longer solvation times, potentially because more extensive solvent rearrangement is required to relax states with lower binding energy.

\section{ASSOCIATED CONTENT}

\section{SI Supporting Information}

The Supporting Information is available free of charge at https://pubs.acs.org/doi/10.1021/acs.jpca.0c06935.

Details on the experiment and data analysis (PDF)

\section{AUTHOR INFORMATION}

\section{Corresponding Author}

Ruth Signorell - ETH Zurich, Department of Chemistry and Applied Biosciences, CH-8093 Zurich, Switzerland; ○ orcid.org/0000-0003-1111-9261; Email: rsignorell@ ethz.ch

\section{Authors}

Loren Ban - ETH Zurich, Department of Chemistry and Applied Biosciences, CH-8093 Zurich, Switzerland

Christopher W. West - ETH Zurich, Department of Chemistry and Applied Biosciences, CH-8093 Zurich, Switzerland

Egor Chasovskikh - ETH Zurich, Department of Chemistry and Applied Biosciences, CH-8093 Zurich, Switzerland

Thomas E. Gartmann - ETH Zurich, Department of Chemistry and Applied Biosciences, $\mathrm{CH}-8093$ Zurich, Switzerland

Bruce L. Yoder - ETH Zurich, Department of Chemistry and Applied Biosciences, $\mathrm{CH}-8093$ Zurich, Switzerland

Complete contact information is available at:

https://pubs.acs.org/10.1021/acs.jpca.0c06935

\section{Notes}

The authors declare no competing financial interest. Data required to reproduce the results presented in the paper can be found in the open access data collection of ETHZ at https://doi.org/10.3929/ethz-b-000438331.

\section{ACKNOWLEDGMENTS}

We thank David Stapfer and Markus Steger for technical support. This project has received funding from the European
Union's Horizon 2020 research and innovation program from the European Research Council under the Grant Agreement No 786636, and the research was supported by the NCCR MUST, funded by the Swiss National Science Foundation (SNSF), through ETH-FAST, and through SNSF project no. 200020 172472. C.W.W. acknowledges funding from the European Union's Horizon 2020 research and innovation programme under the Marie Skłodowska-Curie grant agreement No 801459 - FP-RESOMUS - and the Swiss National Science Foundation through the NCCR MUST. R.S. is a grateful recipient of a Humboldt Research Prize form the Alexander von Humboldt Foundation and a Mildred Dresselhaus Guestprofessorship from the Centre for Ultrafast Imaging in Hamburg.

\section{REFERENCES}

(1) Yamamoto, Y.; Suzuki, Y.-I.; Tomasello, G.; Horio, T.; Karashima, S.; Mitríc, R.; Suzuki, T. Time- and Angle-Resolved Photoemission Spectroscopy of Hydrated Electrons Near a Liquid Water Surface. Phys. Rev. Lett. 2014, 112, 187603.

(2) Yamamoto, Y.-I. I.; Karashima, S.; Adachi, S.; Suzuki, T. Wavelength Dependence of UV Photoemission from Solvated Electrons in Bulk Water, Methanol, and Ethanol. J. Phys. Chem. A 2016, 120, 1153-1159.

(3) Elles, C. G.; Jailaubekov, A. E.; Crowell, R. A.; Bradforth, S. E. Excitation-Energy Dependence of the Mechanism for Two-Photon Ionization of Liquid $\mathrm{H}_{2} \mathrm{O}$ and $\mathrm{D}_{2} \mathrm{O}$ from 8.3 to $12.4 \mathrm{eV}$. J. Chem. Phys. 2006, 125, 044515 .

(4) Young, R. M.; Neumark, D. M. Dynamics of Solvated Electrons in Clusters. Chem. Rev. 2012, 112, 5553-5577.

(5) Chen, X.; Bradforth, S. E. The Ultrafast Dynamics of Photodetachment. Annu. Rev. Phys. Chem. 2008, 59, 203-231.

(6) Bragg, A. E.; Verlet, J. R. R. R.; Kammrath, A.; Cheshnovsky, O.; Neumark, D. M. Electronic Relaxation Dynamics of Water Cluster Anions. J. Am. Chem. Soc. 2005, 127, 15283-15295.

(7) Griffin, G. B.; Young, R. M.; Ehrler, O. T.; Neumark, D. M. Electronic Relaxation Dynamics in Large Anionic Water Clusters: $\left(\mathrm{H}_{2} \mathrm{O}\right)_{\mathrm{n}^{-}}$and $\left(\mathrm{D}_{2} \mathrm{O}\right)_{\mathrm{n}}{ }^{-}(\mathrm{N}=25-200)$. J. Chem. Phys. 2009, 131, 194302.

(8) Savolainen, J.; Uhlig, F.; Ahmed, S.; Hamm, P.; Jungwirth, P. Direct Observation of the Collapse of the Delocalized Excess Electron in Water. Nat. Chem. 2014, 6, 697-701.

(9) Herbert, J. M.; Coons, M. P. The Hydrated Electron. Annu. Rev. Phys. Chem. 2017, 68, 447.

(10) Uhlig, F.; Marsalek, O.; Jungwirth, P. Electron at the Surface of Water: Dehydrated or Not? J. Phys. Chem. Lett. 2013, 4, 338-343.

(11) Casey, J. R.; Schwartz, B. J.; Glover, W. J. Free Energies of Cavity and Noncavity Hydrated Electrons Near the Instantaneous Air/Water Interface. J. Phys. Chem. Lett. 2016, 7, 3192-3198.

(12) Borgis, D.; Rossky, P. J.; Turi, L. Electronic Excited State Lifetimes of Anionic Water Clusters: Dependence on Charge Solvation Motif. J. Phys. Chem. Lett. 2017, 8, 2304-2309.

(13) Horio, T.; Shen, H.; Adachi, S.; Suzuki, T. Photoelectron Spectra of Solvated Electrons in Bulk Water, Methanol, and Ethanol. Chem. Phys. Lett. 2012, 535, 12-16.

(14) Coons, M. P.; You, Z. Q.; Herbert, J. M. The Hydrated Electron at the Surface of Neat Liquid Water Appears to Be Indistinguishable from the Bulk Species. J. Am. Chem. Soc. 2016, 138, 10879-10886.

(15) Stähler, J.; Deinert, J.-C. C.; Wegkamp, D.; Hagen, S.; Wolf, M. Real-Time Measurement of the Vertical Binding Energy during the Birth of a Solvated Electron. J. Am. Chem. Soc. 2015, 137, 3520-3524.

(16) Sagar, D. M.; Bain, C. D.; Verlet, J. R. R. Hydrated Electrons at the Water/Air Interface. J. Am. Chem. Soc. 2010, 132, 6917-6919.

(17) Nowakowski, P. J.; Woods, D. A.; Verlet, J. R. R. Charge Transfer to Solvent Dynamics at the Ambient Water/Air Interface. J. Phys. Chem. Lett. 2016, 7, 4079-4085. 
(18) Elkins, M. H.; Williams, H. L.; Neumark, D. M. Isotope Effect on Hydrated Electron Relaxation Dynamics Studied with TimeResolved Liquid Jet Photoelectron Spectroscopy. J. Chem. Phys. 2016, 144, 184503.

(19) Riley, J. W.; Wang, B.; Woodhouse, J. L.; Assmann, M.; Worth, G. A.; Fielding, H. H. Unravelling the Role of an Aqueous Environment on the Electronic Structure and Ionization of Phenol Using Photoelectron Spectroscopy. J. Phys. Chem. Lett. 2018, 9, 678682.

(20) Coe, J. V.; Arnold, S. T.; Eaton, J. G.; Lee, G. H.; Bowen, K. H. Photoelectron Spectra of Hydrated Electron Clusters: Fitting Line Shapes and Grouping Isomers. J. Chem. Phys. 2006, 125, 14315.

(21) Ma, L.; Majer, K.; Chirot, F.; Von Issendorff, B. Low Temperature Photoelectron Spectra of Water Cluster Anions. J. Chem. Phys. 2009, 131, 144303.

(22) Ehrler, O. T.; Neumark, D. M. Dynamics of Electron Solvation in Molecular Clusters. Acc. Chem. Res. 2009, 42, 769-777.

(23) Lietard, A.; Verlet, J. R. R. R. Selectivity in Electron Attachment to Water Clusters. J. Phys. Chem. Lett. 2019, 10, 1180-1184.

(24) Faubel, M.; Siefermann, K. R.; Liu, Y.; Abel, B. Ultrafast Soft XRay Photoelectron Spectroscopy at Liquid Water Microjets. Acc. Chem. Res. 2012, 45, 120-130.

(25) Turi, L.; Rossky, P. J. Theoretical Studies of Spectroscopy and Dynamics of Hydrated Electrons. Chem. Rev. 2012, 112, 5641-5674.

(26) Kambhampati, P.; Son, D. H.; Kee, T. W.; Barbara, P. F. Solvation Dynamics of the Hydrated Electron Depends on Its Initial Degree of Electron Delocalization. J. Phys. Chem. A 2002, 106, 23742378.

(27) Lian, R.; Oulianov, D. A.; Shkrob, I. A.; Crowell, R. A. Geminate Recombination of Electrons Generated by Above-the-Gap $(12.4 \mathrm{eV})$ Photoionization of Liquid Water. Chem. Phys. Lett. 2004, 398, 102-106.

(28) Kratz, S.; Torres-Alacan, J.; Urbanek, J.; Lindner, J.; Vöhringer, P. Geminate Recombination of Hydrated Electrons in Liquid-toSupercritical Water Studied by Ultrafast Time-Resolved Spectroscopy. Phys. Chem. Chem. Phys. 2010, 12, 12169.

(29) Paik, D. H.; Lee, I. R.; Yang, D. S.; Baskin, J. S.; Zewail, A. H. Electrons in Finite-Sized Water Cavities: Hydration Dynamics Observed in Real Time. Science 2004, 306, 672-675.

(30) Wilhelm, J.; VandeVondele, J.; Rybkin, V. V. Dynamics of the Bulk Hydrated Electron from Many-Body Wave-Function Theory. Angew. Chem., Int. Ed. 2019, 58, 3890-3893.

(31) Luckhaus, D.; Yamamoto, Y.; Suzuki, T.; Signorell, R. Genuine Binding Energy of the Hydrated Electron. Sci. Adv. 2017, 3, No. e1603224.

(32) Zeuch, T.; Buck, U. Sodium Doped Hydrogen Bonded Clusters: Solvated Electrons and Size Selection. Chem. Phys. Lett. 2013, 579, 1-10.

(33) West, A. H. C.; Yoder, B. L.; Luckhaus, D.; Saak, C. M.; Doppelbauer, M.; Signorell, R. Angle-Resolved Photoemission of Solvated Electrons in Sodium-Doped Clusters. J. Phys. Chem. Lett. 2015, 6, 1487-1492.

(34) Pizzochero, M.; Ambrosio, F.; Pasquarello, A. Picture of the Wet Electron: A Localized Transient State in Liquid Water. Chem. Sci. 2019, 10, 7442-7448.

(35) Siefermann, K. R.; Liu, Y.; Lugovoy, E.; Link, O.; Faubel, M.; Buck, U.; Winter, B.; Abel, B. Binding Energies, Lifetimes and Implications of Bulk and Interface Solvated Electrons in Water. Nat. Chem. 2010, 2, 274-279.

(36) Signorell, R.; Yoder, B. L.; West, A. H. C. C.; Ferreiro, J. J.; Saak, C.-M. M. Angle-Resolved Valence Shell Photoelectron Spectroscopy of Neutral Nanosized Molecular Aggregates. Chem. Sci. 2014, 5, 1283-1295.

(37) Madsen, D.; Thomsen, C. L.; Thøgersen, J.; Keiding, S. R. Temperature Dependent Relaxation and Recombination Dynamics of the Hydrated Electron. J. Chem. Phys. 2000, 113, 1126-1134.

(38) Bartels, D. M.; Crowell, R. A. Photoionization Yield vs Energy in $\mathrm{H}_{2} \mathrm{O}$ and $\mathrm{D}_{2} \mathrm{O}$. J. Phys. Chem. A 2000, 104, 3349-3355.
(39) Crowell, R. A.; Bartels, D. M. Multiphoton Ionization of Liquid Water with 3.0-5.0eV Photons. J. Phys. Chem. 1996, 100, 1794017949.

(40) Pépin, C.; Goulet, T.; Houde, D.; Jay-Gerin, J. P. Observation of a Continuous Spectral Shift in the Solvation Kinetics of Electrons in Neat Liquid Deuterated Water. J. Phys. Chem. A 1997, 101, 43514360.

(41) Loh, Z. H.; et al. Observation of the Fastest Chemical Processes in the Radiolysis of Water. Science 2020, 367, 179-182.

(42) Lan, J.; Kapil, V.; Gasparotto, P.; Ceriotti, M.; Iannuzzi, M.; Rybkin, V. V. Simulating the Ghost: Quantum Dynamics of the Solvated Electron. 2020. DOI: 10.26434/chemrxiv.12551234.v1.

(43) Trabattoni, A.; et al. Photoelectron Spectroscopy of Large Water Clusters Ionized by an XUV Comb. J. Phys. Photonics 2020, 2, 035007.

(44) Lübcke, A.; Buchner, F.; Heine, N.; Hertel, I. V.; Schultz, T. Time-Resolved Photoelectron Spectroscopy of Solvated Electrons in Aqueous NaI Solution. Phys. Chem. Chem. Phys. 2010, 12, 1462914634.

(45) Buchner, F.; Schultz, T.; Lübcke, A. Solvated Electrons at the Water-Air Interface: Surface versus Bulk Signal in Low Kinetic Energy Photoelectron Spectroscopy. Phys. Chem. Chem. Phys. 2012, 14, 5837.

(46) Shreve, A. T.; Elkins, M. H.; Neumark, D. M. Photoelectron Spectroscopy of Solvated Electrons in Alcohol and Acetonitrile Microjets. Chem. Sci. 2013, 4, 1633-1639.

(47) Elkins, M. H.; Williams, H. L.; Shreve, A. T.; Neumark, D. M. Relaxation Mechanism of the Hydrated Electron. Science 2013, 342, 1496-1499.

(48) Karashima, S.; Yamamoto, Y. I.; Suzuki, T. Resolving Nonadiabatic Dynamics of Hydrated Electrons Using Ultrafast Photoemission Anisotropy. Phys. Rev. Lett. 2016, 116, 137601.

(49) Gartmann, T. E.; Ban, L.; Yoder, B. L.; Hartweg, S.; Chasovskikh, E.; Signorell, R. Relaxation Dynamics and Genuine Properties of the Solvated Electron in Neutral Water Clusters. J. Phys. Chem. Lett. 2019, 10, 4777-4782.

(50) Yamamoto, Y.; Suzuki, T. Ultrafast Dynamics of Water Radiolysis: Hydrated Electron Formation, Solvation, Recombination, and Scavenging. J. Phys. Chem. Lett. 2020, 11, 5510-5516.

(51) Svoboda, V.; Michiels, R.; LaForge, A. C.; Med, J.; Stienkemeier, F.; Slavíček, P.; Wörner, H. J. Real-Time Observation of Water Radiolysis and Hydrated Electron Formation Induced by Extreme-Ultraviolet Pulses. Sci. Adv. 2020, 6, No. eaaz0385.

(52) Thomsen, C. L.; Madsen, D.; Keiding, S. R.; Thøgersen, J.; Christiansen, O. Two-Photon Dissociation and Ionization of Liquid Water Studied by Femtosecond Transient Absorption Spectroscopy. J. Chem. Phys. 1999, 110, 3453-3462.

(53) Winter, B.; Weber, R.; Widdra, W.; Dittmar, M.; Faubel, M.; Hertel, I. V. Full Valence Band Photoemission from Liquid Water Using EUV Synchrotron Radiation. J. Phys. Chem. A 2004, 108, $2625-2632$

(54) Chen, W.; Ambrosio, F.; Miceli, G.; Pasquarello, A. Ab Initio Electronic Structure of Liquid Water. Phys. Rev. Lett. 2016, 117, 186401.

(55) Ambrosio, F.; Guo, Z.; Pasquarello, A. Absolute Energy Levels of Liquid Water. J. Phys. Chem. Lett. 2018, 9, 3212-3216.

(56) Gaiduk, A. P.; Pham, T. A.; Govoni, M.; Paesani, F.; Galli, G. Electron Affinity of Liquid Water. Nat. Commun. 2018, 9, 247.

(57) Vilchiz, V. H.; Kloepfer, J. A.; Germaine, A. C.; Lenchenkov, V. A.; Bradforth, S. E. Map for the Relaxation Dynamics of Hot Photoelectrons Injected into Liquid Water via Anion Threshold Photodetachment and above Threshold Solvent Ionization. J. Phys. Chem. A 2001, 105, 1711-1723.

(58) Nishitani, J.; Yamamoto, Y.; West, C. W.; Karashima, S.; Suzuki, T. Binding Energy of Solvated Electrons and Retrieval of True UV Photoelectron Spectra of Liquids. Sci. Adv. 2019, 5, No. eaaw6896.

(59) Hertwig, A.; Hippler, H.; Unterreiner, A. N. Transient Spectra, Formation, and Geminate Recombination of Solvated Electrons in Pure Water UV-Photolysis: An Alternative View. Phys. Chem. Chem. Phys. 1999, 1, 5633-5642. 
(60) Mozumder, A. Conjecture on Electron Trapping in Liquid Water. Int. J. Radiat. Appl. Instrumentation. Part C. Radiat. Phys. Chem. 1988, 32, 287-291.

(61) Signorell, R. Electron Scattering in Liquid Water and Amorphous Ice: A Striking Resemblance. Phys. Rev. Lett. 2020, 124, 205501.

(62) Michaud, M.; Wen, A.; Sanche, L. Cross Sections for LowEnergy $(1-100 \mathrm{eV})$ Electron Elastic and Inelastic Scattering in Amorphous Ice. Radiat. Res. 2003, 159, 3-22.

(63) Signorell, R.; Goldmann, M.; Yoder, B. L.; Bodi, A.; Chasovskikh, E.; Lang, L.; Luckhaus, D. Nanofocusing, Shadowing, and Electron Mean Free Path in the Photoemission from Aerosol Droplets. Chem. Phys. Lett. 2016, 658, 1-6.

(64) Gartmann, T. E.; Hartweg, S.; Ban, L.; Chasovskikh, E.; Yoder, B. L.; Signorell, R. Electron Scattering in Large Water Clusters from Photoelectron Imaging with High Harmonic Radiation. Phys. Chem. Chem. Phys. 2018, 20, 16364-16371.

(65) Yoder, B. L.; West, A. H. C. C.; Schläppi, B.; Chasovskikh, E.; Signorell, R. A Velocity Map Imaging Photoelectron Spectrometer for the Study of Ultrafine Aerosols with a Table-Top VUV Laser and NaDoping for Particle Sizing Applied to Dimethyl Ether Condensation. J. Chem. Phys. 2013, 138, 44202.

(66) Yoder, B. L.; Litman, J. H.; Forysinski, P. W.; Corbett, J. L.; Signorell, R. Sizer for Neutral Weakly Bound Ultrafine Aerosol Particles Based on Sodium Doping and Mass Spectrometric Detection. J. Phys. Chem. Lett. 2011, 2, 2623-2628.

(67) Baumann, A.; et al. Weak-Field Few-Femtosecond VUV Photodissociation Dynamics of Water Isotopologues. Phys. Rev. A: At., Mol., Opt. Phys. 2017, 96, 013428.

(68) van Stokkum, I. H. M.; Larsen, D. S.; van Grondelle, R. Global and Target Analysis of Time-Resolved Spectra. Biochim. Biophys. Acta, Bioenerg. 2004, 1657, 82-104.

(69) Hara, A.; Yamamoto, Y. I.; Suzuki, T. Solvated Electron Formation from the Conduction Band of Liquid Methanol: Transformation from a Shallow to Deep Trap State. J. Chem. Phys. 2019, 151, 114503.

(70) Mosyak, A. A.; Prezhdo, O. V.; Rossky, P. J. Solvation Dynamics of an Excess Electron in Methanol and Water. J. Chem. Phys. 1998, 109, 6390-6395.

(71) Signorell, R. Can Current Experimental Data Exclude NonGaussian Genuine Band Shapes in Ultraviolet Photoelectron Spectra of the Hydrated Electron? J. Phys. Chem. Lett. 2020, 11, 1516-1519.

(72) Hartweg, S.; Yoder, B. L.; Garcia, G. A.; Nahon, L.; Signorell, R. Size-Resolved Photoelectron Anisotropy of Gas Phase Water Clusters and Predictions for Liquid Water. Phys. Rev. Lett. 2017, 118, 103402. 